\title{
Effects of Hardness of Primordial Binaries on Evolution of Star Clusters
}

\author{
A. Tanikawa ${ }^{1}$ and T. Fukushige ${ }^{2}$ \\ ${ }^{1}$ Department of General System Studies, College of Arts and Sciences, University of Tokyo \\ email: tanikawa@ea.c.u-tokyo.ac.jp \\ ${ }^{2}$ K\&F Computing Research Co. \\ email: fukushig@kfcr.jp
}

\begin{abstract}
We performed $N$-body simulations of star clusters with primordial binaries using a new code, GORILLA. It is based on Makino and Aarseth (1992)'s integration scheme on GRAPE, and includes a special treatment for relatively isolated binaries. Using the new code, we investigated effects of hardness of primordial binaries on whole evolution of the clusters. We simulated seven $N=16384$ equal-mass clusters containing 10\% (in mass) primordial binaries whose binding energies are 1,3,10,30,100,300, and $1000 k T$, respectively. Additionally, we also simulated a cluster without primordial binaries and that in which all binaries are replaced by stars with double mass, as references of soft and hard limits, respectively. We found that, in both soft $(\leqslant 3 k T)$ and hard $(\geqslant 1000 k T)$ limits, clusters experiences deep core collapse and shows gravothermal oscillations. On the other hands, in the intermediate hardness $(10-300 k T)$, the core collapses halt halfway due an energy releases of the primordial binaries.
\end{abstract}

Keywords. globular clusters: general, method: n-body simulations

\section{Introduction}

By means of previous numerical simulations, it has been clear that the presence of primordial binaries changes the dynamical evolution of star clusters. The clusters with many primordial binaries experience shallower core collapse than those without primordial binaries by one order of magnitude, and their gravothermal oscillations are delayed by several ten or hundreds half-mass relaxation time (Gao et al. 1991; Fregeau et al. 2003; Giersz \& Spurzem 2003; Heggie et al. 2006). This is due to energy released by the primordial binaries that prevents the clusters from core collapsing.

Many previous works have numerically treated the dynamical evolution of star clusters with primordial binaries by means of $N$-body, Fokker-Planck, and Monte-Carlo simulations. However, they confined binding energy distribution of the primordial binaries to some ranges, and uniform distribution in logarithm of binding energy. There are no special reasons for these distributions. We systematically study the effect of the distributions on cluster evolutions. We set up the simplest binding energy distribution. All binding energies of primordial binaries are equal.

\section{Method}

We investigated the evolution of star clusters by means of $N$-body simulations. We used a new $N$-body simulation code, GORILLA, which we developed. GORILLA is neither based on NBODY (e.g. Aarseth 2003) nor kira (e.g Portegies Zwart et al. 2001), and applies for clusters of point-mass particles. It adopts the fourth-order Hermite integrator with individual timestep algorithm (Makino \& Aarseth 1992, hereafter MA92), 

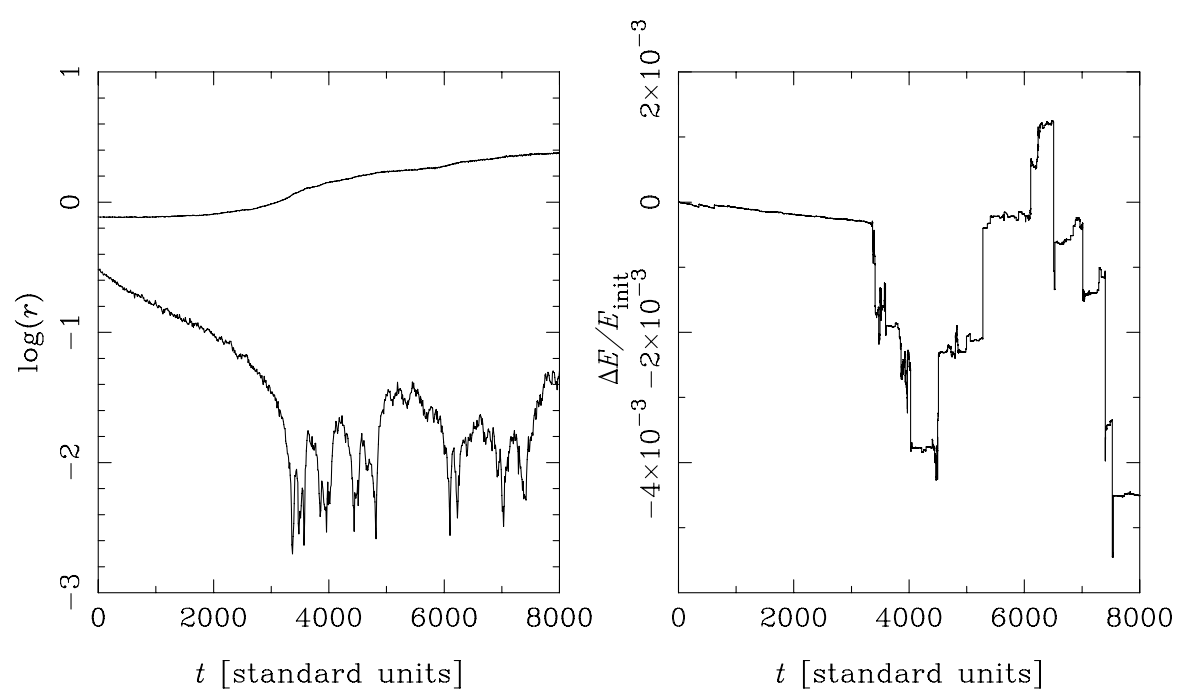

Figure 1. Time evolution of the core, $r_{\mathrm{c}}$, and half-mass radius, $r_{\mathrm{h}}$, (left) and the relative energy error from the initial time, $\Delta E / E_{\text {init }}$, (right) of the cluster without primordial binaries.

and supports GRAPE-6/6A (Makino et al. 2003; Fukushige et al. 2005). Additionally, it is equipped with special treatments for close encounter between two or three particles.

In the special treatment, we approximate the internal motion of a binary as Kepler motion, by neglecting perturbations from other particles. The conditions of the binary are that the binding energy is more than $1 k T$, where $3 / 2 k T$ is the average kinetic energy of cluster stars, and the separation between the binary and the nearest particle are five times more than those between the binary components at the apocenter. If this binary and the nearest particle form a hierarchical triple system, in which the binary (hereafter "inner binary") and the nearest particle revolve around each other (hereafter "outer binary"), we approximate the internal motion between the center of mass of the inner binary, and the nearest particle as Kepler motion. The condition is that the binding energies of the inner and outer binary are more than $1 k T$, the separation between the inner binary and the particle at the pericenter is five times more than that between the inner binary components at the apocenter, and the separation between the hierarchical triple system and the nearest particle is five times more than that between the inner binary and the particle at the apocenter.

We performed the test simulation of GORILLA. In the test simulation, we adopted a cluster with $N=16384$ equal-mass and single stars, and the standard units. Fig. 1 shows the time evolution of the core and half-mass radii (left panel), and the relative energy error from the initial time (right panel) of the cluster. GORILLA can follow the evolution of the cluster from gravothermal core collapse to gravothermal oscillations. The energy error is less than $0.5 \%$ of the initial total energy.

By means of GORILLA, we performed $N$-body simulations of clusters with many primordial binaries. We also adopted the standard units. The initial distribution function is Plummer model. The number of particles is 16384, and they are equal-mass. These clusters contain $10 \%$ primordial binaries in mass. The primordial binaries have equal binding energies in each model, and eccentricity with thermal distribution, $f(e)=2 e$, where $e$ is eccentricity. We have 9 clusters with different binding energies, no binaries (in other words $0 k T$ ) $, 1,3,10,30,100,300$, and $1000 k T$, and double mass stars (in other 


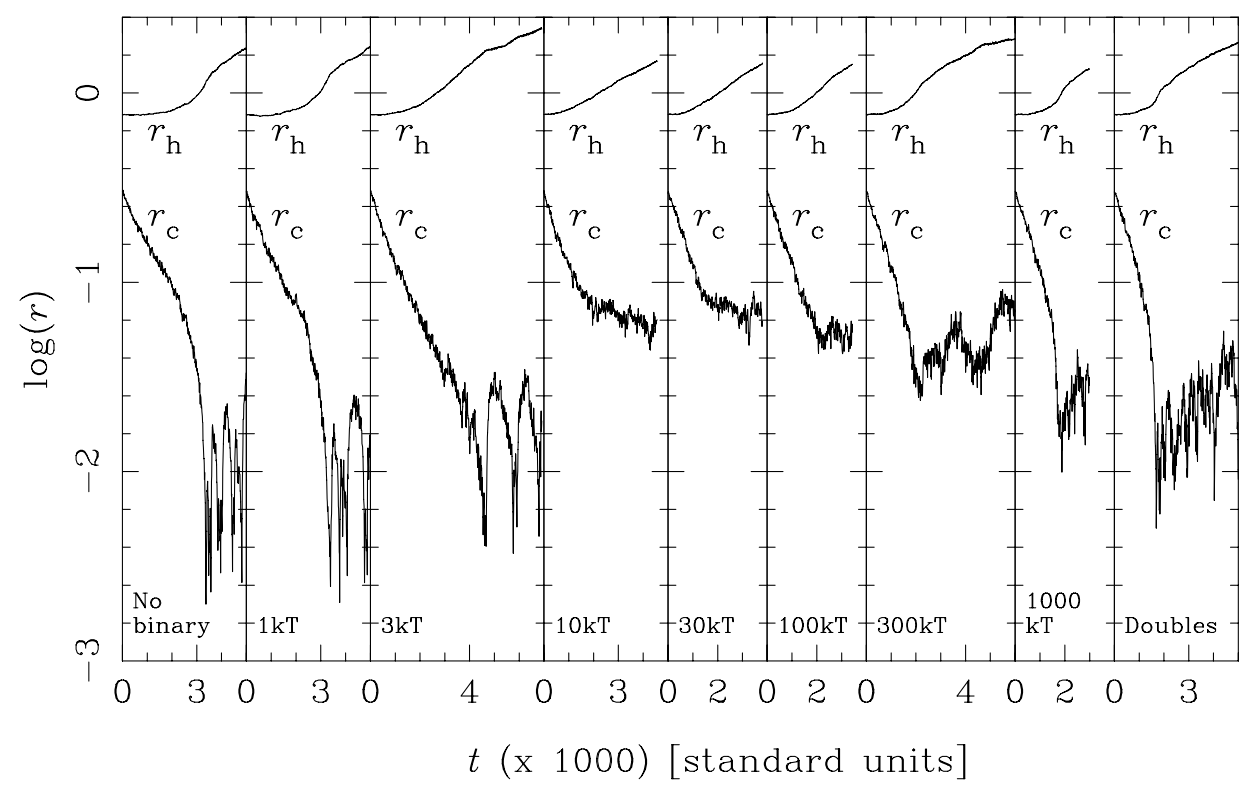

Figure 2. Time evolution of the core, $r_{\mathrm{c}}$, and half-mass radii, $r_{\mathrm{h}}$, of the clusters without primordial binaries, with $1,3,10,30,100,300$, and $1000 k T$ primordial binaries, and with double mass stars, from left panel to right panel.

words $\infty k T)$. We call these clusters "No binary", $1-1000 k T$, and "Doubles" clusters, respectively. The energy errors in all the simulations are $\sim 0.1-1 \%$.

\section{Results}

Fig. 2 shows the time evolution of the core and half-mass radii of the clusters. The clusters with soft $(\leqslant 3 k T)$, and hard $(\geqslant 1000 k T)$ primordial binaries experienced deep core collapse, $r_{\mathrm{c}} / r_{\mathrm{h}} \sim 10^{-3}-10^{-2}$, and gravothermal oscillations. The clusters with primordial binaries of intermediate hardness $(10-300 k T)$ experienced shallow core collapse, $r_{\mathrm{c}} / r_{\mathrm{h}} \sim 10^{-2}-10^{-1}$, and steady core evolutions. We define core collapse time as the time when core collapses stop in the case of "No binary", 1, 3, and $1000 k T$, and "doubles" clusters, and the time when core collapses slow down in the case of $10-300 k T$ clusters. The core collapse time of $3 k T$ is more delayed than that of $1 k T$ cluster. $300 k T$ cluster seems to show gravothermal oscillations. This cluster shows the intermediate behavior between 100 and $1000 k T$.

The difference of the evolutions results from released energy by primordial binaries. Fig. 3 shows the total energies released by binaries from the initial times. In the case of $1 k T$ cluster, the binaries release little energy before core collapse. In the case of $3 k T$ cluster, the binaries release steadily halfway. This is due to the more delayed core collapse time than that of $1 k T$ cluster. In the cases of $10-300 k T$ clusters, the binaries release energy steadily from the beginning. Despite of large energy released in $1000 k T$ cluster, the cluster experiences the deep core collapse. When a $1000 k T$ binary encounters with other stars, this binary releases so much energy that they can escape from the cluster on crossing timescale. Therefore, the energy released by the $1000 k T$ binaries is carried away by the binaries and stars involved in the encounters.

Fig. 4 show the time evolution of the number of binaries. Binaries of $1 k T$ cluster decrease more rapidly than those in the cases of $10-1000 k T$. This is due to little energy released by $1 k T$ binaries. 


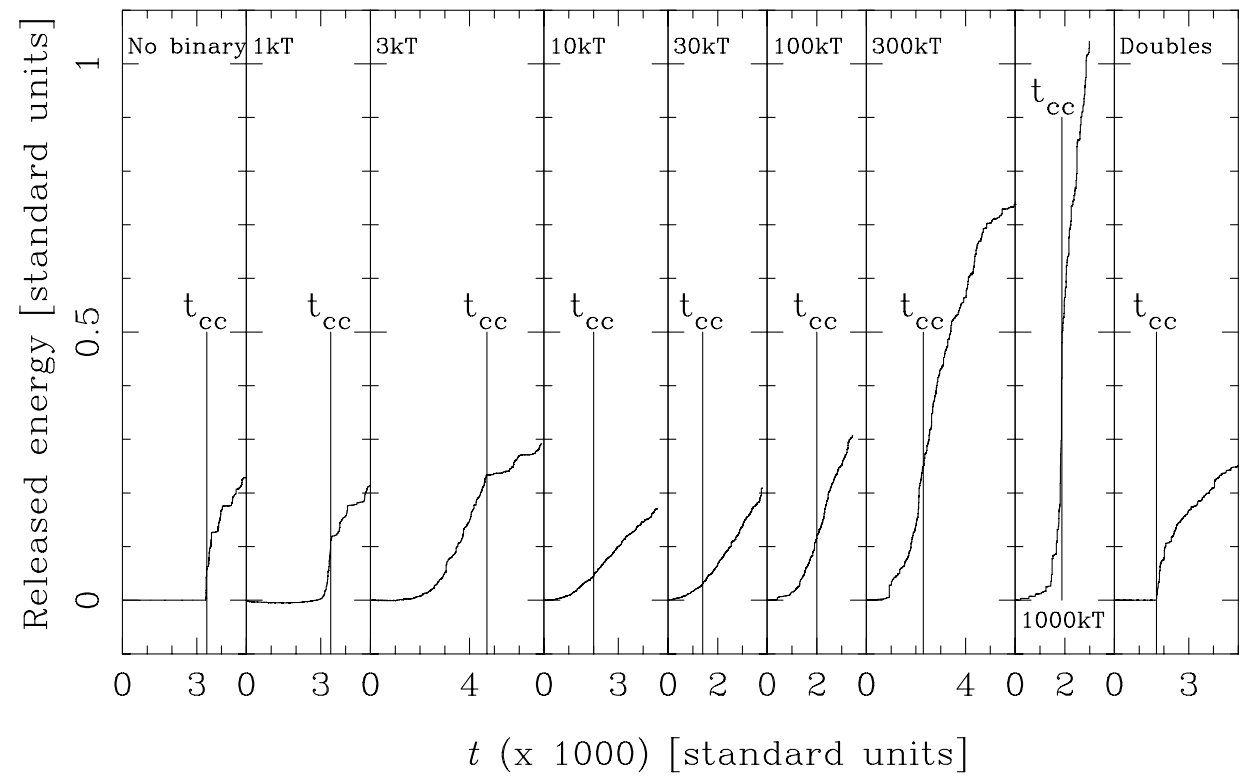

Figure 3. Time evolution of total energy released by binaries from initial time. Vertical lines show core collapse times, $t_{\mathrm{cc}}$.

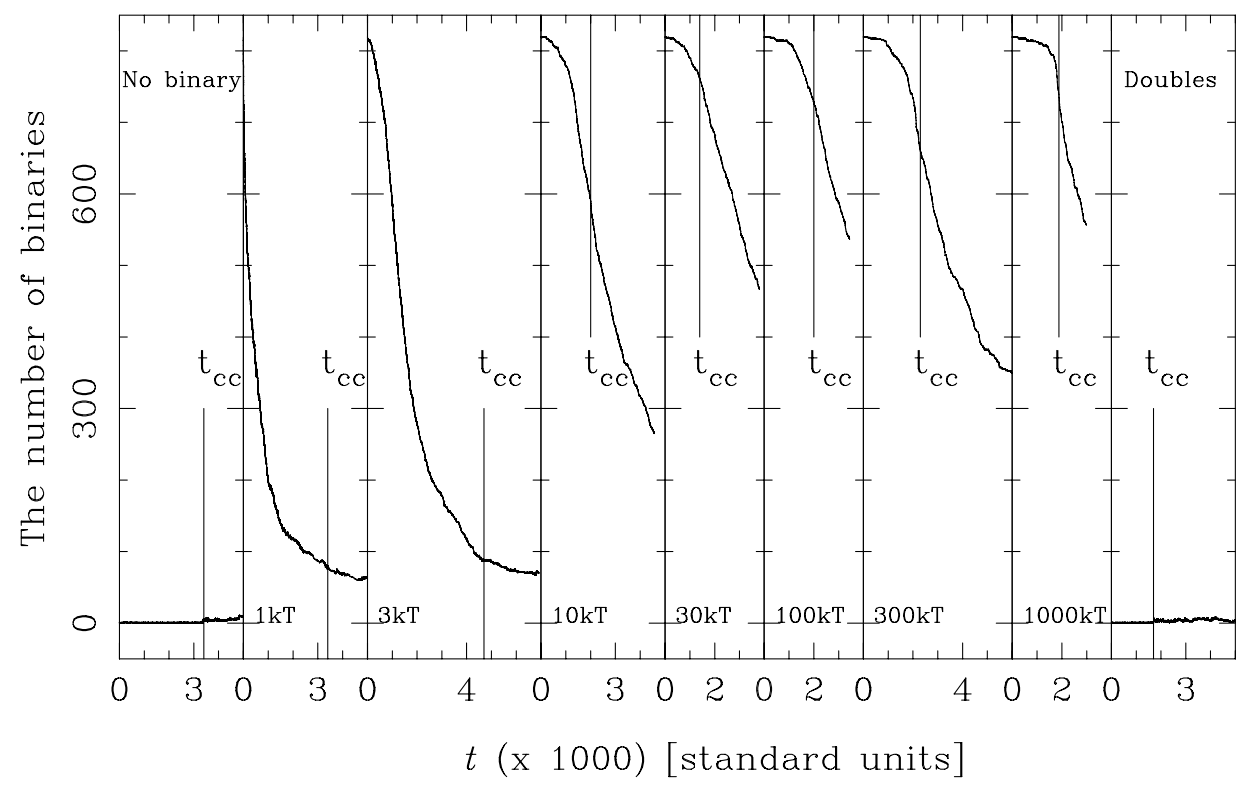

Figure 4. Time evolution of the number of binaries. Vertical lines show core collapse times,

\section{Summary}

We developed a new $N$-body simulation code, GORILLA. By means of GORILLA, we investigated the evolution of star clusters with $10 \%$ primordial binaries in mass. We followed the evolution of nine sets of clusters that each have primordial binaries with equal binding energy.

The clusters experienced deep core collapses in soft $(\leqslant 3 k T)$ and hard $(\geqslant 1000 k T)$ limits, and shallow core collapses in the intermediate region of hardness $(10-300 k T)$. 
The reasons are as follows. In $\leqslant 3 k T$, little energy is released due to the rapid destruction. In $10-300 k T$, energy is steadily released. In $\geqslant 1000 k T$, released energy is carried away by the binary itself and stars involving the encounter. The fact shows that only primordial binaries with $10-300 k T$ are effective sources of star clusters.

\section{References}

Aarseth, S. 2003, Gravitational $N$-body Simulations (Cambridge:Cambridge University Press) CHW89] Cohn, H., Hut, P., \& Wise, M. 1989, ApJ, 342, 814

Fregeau, J. M., Gurkan, M. A., Joshi, K. J., \& Rasio, F. A. 2003, ApJ, 593, 772

Fukushige, T., Makino, J., \& Kawai, A. 2005, PASJ, 57, 1009

Gao, B., Goodman, J., Cohn, H., \& Murphy, B. 1991, ApJ, 370, 567

Giersz, M. \& Spurzem, R. 2003, MNRAS, 343, 781

Heggie, D. C. $1975, M N R A S, 173,729$

Heggie, D. C. \& Mathieu, R. D. 1986, in Lecture Notes in Physics Vol. 267, ed. P. Hut \& S. McMillan (Berlin: Springer-Verlag), 233

Heggie, D. C., Trenti, M., \& Hut, P. 2006, ApJ, 368, 677

Makino, J. \& Aarseth, S. 1992, PASJ, 44, 141

Makino, J., Fukushige, T., Koga, M., \& Narumi, K. 2003, PASJ, 55, 1163

Portegies Zwart, S. F., McMillan, S. L. W., Hut, P., \& Makino, J. 2001 MNRAS, 321, 199

Vespereini, E. \& Chernoff, D. F. 1994, ApJ, 431, 231 